\title{
YIELD FROM NEUROIMAGING IN MIGRAINE
}

\author{
S. Sampath, D. Pintus, L. Lekkala, C. Gillon, F. Godinho, M. Aziz, C. John
}

St. Helens Knowsley NHS Trust, Prescot, UK

Introduction: Increasing numbers of children with chronic headaches are referred from primary care due to uncertainty of diagnosis or for symptom control. Although the diagnosis of migraine is essentially clinical, neuroimaging may be performed if there are worrying neurological symptoms or signs, or poor response to treatment.

Aim: To determine the indications and yield from neuroimaging in migraine.

Methods: We identified 31 children (19 boys and 12 girls) with migraine who either had Magnetic resonance imaging (MRI) or computed tomography (CT) scans of the brain. Mean age at onset of migraine symptoms was $11.8 \pm 2.56$ (7-16) yrs. Mean age at neuroimaging was $12.9 \pm 3.03$ (8-17) yrs. The indications for neuroimaging could be categorised as follows: increasing frequency or severity of headaches $(n=12)$ reported vision abnormality $(n=5)$, suspected raised intracranial pressure $(n=5)$ co-existent epilepsy $(n=4 L)$ 'dizzy /collapse' $(n=2)$ and other neurological symptoms $(n=3)$. The MRI scans were abnormal in 7 of 28 cases. Abnormalities included sinusitis in four, demyelination in one, distorted anatomy of cochlea/vestibule in one and medial temporal lobe sclerosis in one. CT scan in one of three cases revealed sinusits. Following negative neuroimaging $5 / 23$ had dramatic improvement of symptoms which facilitated discharge from clinic.

Conclusion: Radiological evidence of sinusitis co-existed in 5 children with migraine but was suspected in only one patient. It is important to consider sinusitis in chronic headaches. Normal neuroimaging may relieve parental anxiety and we speculate that this aided resolution of symptoms and facilitated discharge from clinic in $5 / 31$ patients. 